\title{
Strategy for monitoring and ensuring safe operation of Russian gas transportation systems
}

\author{
Sergey Dudin ${ }^{1}$, Gennady Bahmat ${ }^{1}$, Yury Zemenkov ${ }^{1}$, Konstantin Voronin ${ }^{1}$, and Anton \\ Shipovalov ${ }^{2, *}$ \\ ${ }^{1}$ Industrial University of Tyumen, 38 Volodarskogo st, Tyumen, 625000, Russia \\ ${ }^{2}$ Gazprom TransgazYugorsk, Yugorsk, 15 Mira st, 628260, Russia
}

\begin{abstract}
The authors examined the legislative framework of the Russian Federation operating in the field of industrial safety, and described how to obtain a license to operate hazardous production facilities. The paper demonstrates that the importance should be given to the quality and completeness of the background information, as well as the choice of inspection strategy in evaluating the technical condition of the line section of main gas pipelines. As a part of a package of measures to ensure industrial safety and technical reliability of existing gas pipelines it is proposed to carry out conditioning of their line sections. The paper describes general requirements for pipeline inspection, which include: detection efficiency of dangerous damages and major leaks, maximum accuracy of detection of the defect location, continuity of monitoring throughout the entire range of operation modes of the pipeline, cost recovery at the stages of development and operation of control systems, forecasting the state of the pipeline and the effects of accidents. In practice, these parameters have the prevailing significance.
\end{abstract}

\section{Introduction}

Russian federal legislation in the field of industrial safety includes a number of laws, decrees of the President, resolutions of the Government and competent authorities [1-6 and others].

These documents regulate virtually all aspects of operation of hazardous industrial facilities and related challenges of environmental nature, health protection and creation of safe working conditions. A legislative obligation of legal entities and individuals operating hazardous facilities involves providing risk assessment and underwriting liability. The right to operate hazardous facilities is provided upon receiving a license (permit). Obtaining a

\footnotetext{
*Corresponding author: $\underline{\text { srg_work@mail.ru }}$
} 
license, in turn, involves passing a number of pre-established procedures that can be divided into 3 stages.

The first stage - preparatory:

- Conducting conditioning of the equipment used, assessment of the technical condition of the facilities;

- Assessment of workplaces according to working conditions [1];

- Identification of hazardous production facilities [4];

- Risk analysis of hazardous production facilities [2];

- Entering into the compulsory liability insurance agreement against damage [3];

The first stage ends with the preparation of the declaration of industrial safety of hazardous production facilities.

The second stage - examination of the object. Examination is performed by the organization that has a license in the manner specified in $[5,6]$. In case of a positive conclusion of the examination the third stage takes place - the procedure of obtaining a license $[4,5,6]$. If the conclusion of the examination is negative or if there are comments one should return to the first stage.

From the above it is clear that importance should be given to the quality and completeness of the background information during conditioning and attestation of the technical condition of the entire gas transmission system and, in particular, its line section.

\section{Research object}

In the course of collecting the necessary information about the technical condition of the line section, ie, essentially its diagnosis, serious problems exist. First of all, they are associated with large amounts of diagnostic operations and their technical complexity, and at the first stage of their implementation - also with the right choice of methods and means of diagnosing (inspection). A major scientific problem is the choice of one of inspection strategies.

A complete instrument control strategy comes down to one hundred percent inspection of the entire line section with the help of technical means. In reality, this is only possible with the help of in-line inspection tools, but at the same time information on the status of line valves is incomplete and a separate stage of diagnosis of their state through the use of external control methods is required. This strategy requires considerable financial, organizational and labor costs both at the stage of technical activities, and in the interpretation and analysis of the data obtained.

With the necessary funding the complete instrument control strategy is the most profitable, because it provides a complete picture of the state of the pipe body and welds and a fairly complete picture of the state of other components of the line section. When using this strategy, risk assessment is most accurate.

A mixed evaluation strategy is a combination of computational methods for determining the technical condition and reliability of the line section, followed by a method of selective instrument control diagnosis for clarification and adjustment of mathematical models of the technical condition of the line section.

\section{Methods}

Given a large accumulated experience of creating mathematical models for assessing the reliability and technical condition $[7,8,11,12$ and others] together with the experimental data from instrument control sampling, it can be assumed that the accuracy of the information received will be quite high, although it will retain its probabilistic character. 
Financial costs will significantly decrease compared to the first strategy. The main scientific problem when applying the second strategy is to select and calculate the optimal balance between data arrays obtained experimentally and theoretically, as well as to ensure the adequacy of the final mathematical model taking into account changes in the technical condition and reliability of the line section in time and at varying pumping modes.

Risk assessment when applying this model is less accurate, but sufficient compared to the previous one.

An estimates strategy differs from the previous one in that the instrument control is not performed and refinement of technical condition and reliability mathematical models requires the use of statistical data on failures of the line section and project documentation containing details about pipe metal, quality control of welded joints, passports of valves, soil characteristics, conditions of routing, etc. At the appropriate level of mathematical processing these data will evaluate the probable picture of the line section and the risks associated with its operation with sufficiently high accuracy. Of course, the accuracy of prediction will be inferior to the previous strategy. During subsequent examination such strategy should be theoretically justified at a very high scientific level to ensure that the examination would recognize the results as valid.

These strategies almost completely exhaust the field of choice, although they allow elaboration of other admissible strategies built on real decision-making procedures by expert organizations.

The final choice of strategy depends on the volume of funds, deadlines of work on conditioning and examination of the line section, qualification of involved staff and should be done after the technical and economic analysis at the level of top managers. If the first or the second strategy is selected, it is necessary to determine which methods and diagnostic tools will be used to evaluate the technical condition, reliability and risk of operating the line section.

The current pipeline network is the leader among all kinds of transport in performance, product turnover, delivery range and cost of pumping hydrocarbon raw materials. Its advantage is that the technical level of domestic pipelines is not inferior to world standards. The total length of Russian gas transportation mains is around 150 thousand $\mathrm{km}$.

The bulk of gas is supplied to the European part of the country from Western Siberia via pipelines with a considerable service life. The life of film insulating coatings of large diameter gas pipelines (about $50 \%$ of all pipes) comes to its critical date, which significantly increases the likelihood of accidents on the line section of main pipelines as a result of corrosion damage to their walls.

When conditioning the line section of main gas pipelines there is a serious problem of choosing (ranking) the sections the diagnosis of which is to be held in the first place and with the greatest accuracy. Solution of this problem will allow for a more reasonable selection of one of the strategies of evaluation of the line section technical condition, reduction of the amount of work during diagnosis and improvement in the reliability of the line section of main gas lines while reducing risks.

Analysis of accidents on main gas pipelines in Western Siberia allows us to propose a ranking of the line sections according to their risks depending on the distance from the compressor station (CS) (pressure in the pipe), laying category, insulation coating category, location of line sections [9, 10]. Distribution of accidents by distance from the CS is represented as a diagram in Fig. 1. 


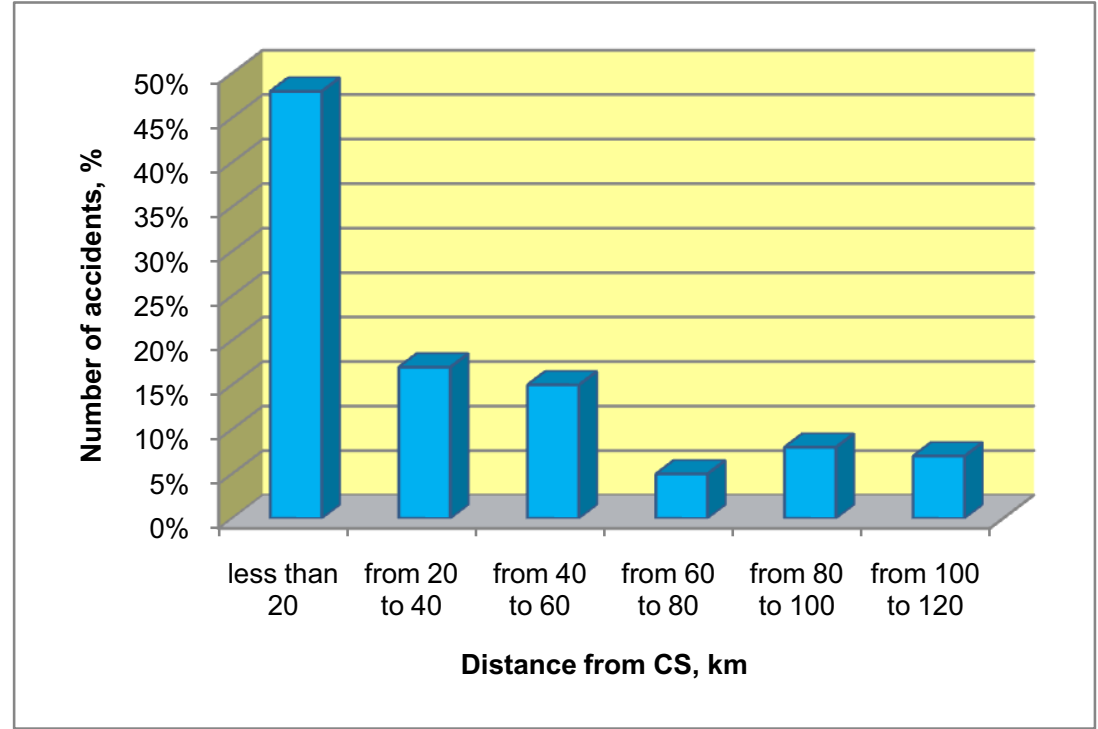

Fig. 1.Ranking of the number of accidents (in \%) depending on the distance

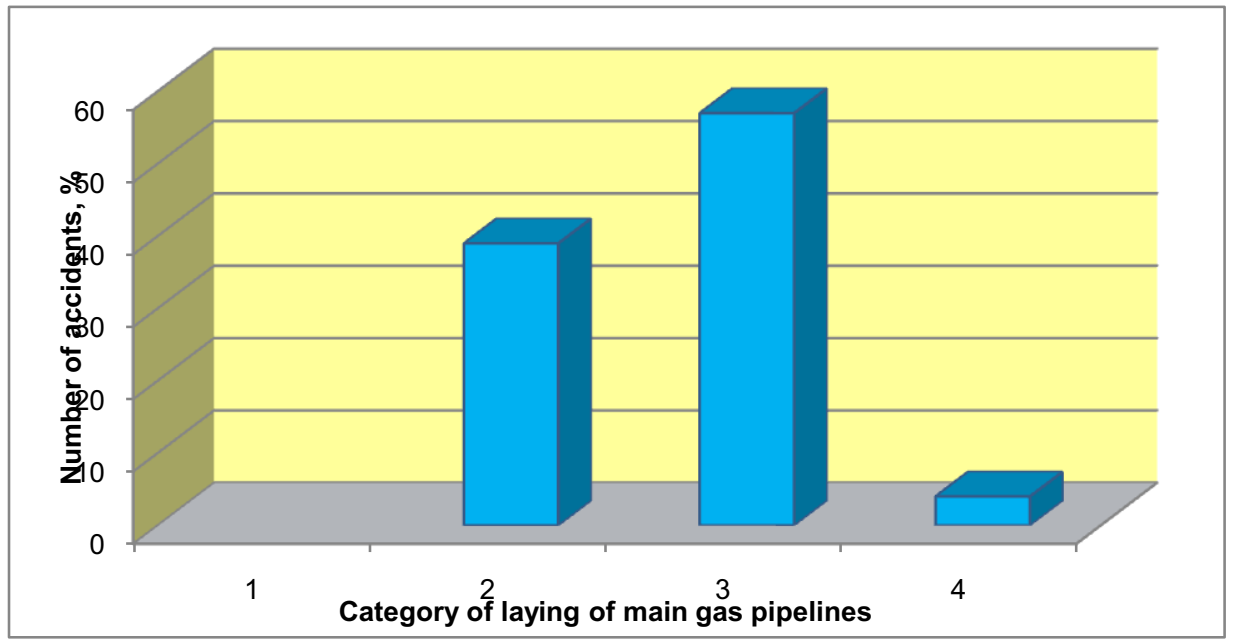

Fig. 2.Percentage ratio of the number of accidents depending on the categories of gas pipeline routes

It is clear from this diagram that approximately $50 \%$ of all accidents occurred at a distance of $20 \mathrm{~km}$ from the CS in the direction of gas movement. At a farther distance from the CS the number of accidents decreases from $17 \%$ for $20<\mathrm{x}<40$, to $7 \%$ for $100<\mathrm{x}<$ 120. The implemented analysis of emergency situations based on the location of the emergency point $\mathrm{x}$ along the pipeline route allowed the pipeline to be split into three risk categories:

1. Potentially risky section $(\mathrm{x} \leq 20 \mathrm{~km})$;

2. Risky section $(20<\mathrm{x} \leq 60 \mathrm{~km})$;

3. Low-risk section $(\mathrm{x}>60 \mathrm{~km})$.

The number of accidents by category of laying of main gas pipelines is shown in Fig. 2.

The presented data show that $96 \%$ of all accidents occurred in sections with categories 2 and 3 , which indicates their potential risk.

Thermodynamic conditions are the basis for calculating the reliability of line sections, as well as the basis for forecasting the residual resource of operated pipelines. Of greatest 
interest in this case are sections that are operated in unstable temperature conditions, ie, with positive and negative temperatures throughout the year. Analysis of operation of such sections is particularly important for areas of eternal and sluggish permafrost (northern areas), because in these sections increased stress concentrators are formed substantially reducing their service life.

During conditioning of the line sections of main gas pipelines their stress-strain state must be taken into account. For this purpose it is necessary to carry out land surveying of curved sections which involves measurement of at least 11 points of the curved section.

Next, a mathematical calculation of the stress-strain state is carried out taking into account the elastic, plastic deformation and conditions of corrugation and buckling. Assessing the level of the stress-strain state using strain gauges is irrational because the method is very costly and time-consuming and provides accuracy comparable with the accuracy of calculation methods.

It is important to identify areas where the soil is frozen, permafrost and heaving, and analyze the thermal conditions of the line section in these areas with a view to identifying possible local thermo mechanical stresses.

At the current technical level and taking into account the experience of in-line inspection devices of major oil transport mains for the line sections of main gas lines application of a profile measuring tool is also most effective for magnetic flaw detectors of high resolution.

The use of non-destructive testing methods for the outer surface of the line section of the main gas pipeline in operation is only possible in buried sites and, in part, line valves.

Domestic and foreign experience in the application of different means of control of a technical condition of pipelines in operation shows that the use of microprocessor devices and computers can significantly improve the diagnosis and monitoring systems. When improving instrument inspection systems in pipelines one of the main issues is elimination of operating errors made by service personnel, errors of instrument readings, mathematical methods, means of data collection and transmission, etc.

\section{Results and discussion}

General requirements for pipeline inspection can be formulated as follows: efficiency of detection of dangerous damages and major leaks, maximum accuracy of detection of the defect location, continuity of monitoring throughout the entire range of operation modes of the pipeline, cost recovery at the stages of development and operation of control systems, forecasting the state of the pipeline and the effects of accidents. In practice, these parameters have the prevailing significance. In the foreign literature such factors are also taken into account as the possibility of introducing technological changes in the working schemes, availability on the market, frequency of issuing false signals, amount of maintenance. It should be noted that to some extent the known methods of diagnostics allow us to assess the current state of the pipeline without providing the full required set of technical specifications. Two identical defects located on different pipelines or even on different parts of the same pipeline can cause accidents with a significantly different probability. The same defect at various stages of operation of the transport system can be both acceptable and unacceptable due to changes in the mechanical characteristics of the steel pipe, loading conditions, and other factors. 


\section{Conclusion}

1) Conditioning of the line section of main gas pipelines for subsequent certification and licensing of the hazardous industrial facility - the main gas pipeline is an important part of a set of measures to ensure safety and technical reliability.

2) Analysis of technical condition, reliability and risk of operation of pipelines is impossible without proper selection of the line section control strategy. In relation to the "cost-accuracy" ratio the most acceptable strategy is the strategy of mixed assessment of the line section technical condition.

3) Conditioning requires the use of several methods for instrument monitoring of the technical condition - non-destructive (internal and external) and with the destruction of individual samples. The ratio of frequency of using the methods should be analyzed separately on the basis of economic capabilities.

\section{References}

1. Law "On sanitary and epidemiological welfare of population" of 30.03.99, № 52-FZ.

2. RF GosGorTechNadzor Resolution of 24.08.2001 №37 "Concerning the Implementation of Guidelines for risk analysis of hazardous production facilities".

3. Law "On compulsory social insurance against industrial accidents and occupational diseases" of 29.02.2012, №16-FZ.

4. Law "On industrial safety of hazardous production facilities “of 21.07.97, № 116 FZ (ed. of 25.06.2012).

5. RF Government Resolution "On approval of the Rules of submission of the declaration of industrial safety of hazardous production facilities “of 11.05.99, №529. (amended on 1 February 2005, 22 April 2009, 4 February 2011).

6. Rules of industrial safety expertise. RF GosGorTechNadzor Resolution of 6.10.98, №64, PB 03-246-98. (with changes introduced byDecision of the Supreme Court of RF of 15.05.2008 №GKPI08-1075).

7. N.V.Eremin, O.A.Stepanov, E.I.Yakovlev, Compressor stations of main gas pipelines (Nedra, St.-Petersburg,1995)

8. Yu.D. Zemenkov, Monitoring of hydrodynamic and technical specifications of pipeline systems: Textbook (VektorBuk, Tyumen, 2008)

9. S.M.Dudin, Yu.D.Zemenkov, G.V.Bahmat, V.N.Antipiev, Conditioning as the prospect of industrial safety management system of the line section of main pipelines (TSOGU, Tyumen, 2001)

10. S.M.Dudin, Yu.D.Zemenkov, G.V.Bahmat, Improved safety of operating line sections of pipelines(VNIIST, Moscow, 2012)

11. Yu.D. Zemenkov, Machines and processes in the transport of energy resources: Textbook in two volumes (VektorBuk,Tyumen, 2008)

12. Yu.D. Zemenkov, Operation of main gas pipelines: Textbook (VektorBuk, Tyumen, 2003) 